\title{
A New Solution for the Compression of a Two-Layer Strip and Its Application to Analysis of Bonding by Rolling
}

\author{
Sergei Alexandrov ${ }^{1}$ and Yusof Mustafa ${ }^{2}$ \\ ${ }^{1}$ A.Yu. Ishlinskii Institute for Problems in Mechanics, Russian Academy of Sciences, 101-1 Prospect Vernadskogo, Moscow 119526, Russia \\ ${ }^{2}$ Faculty of Mechanical Engineering, Universiti Teknologi Malaysia, 81310 Skudai, Johor Darul Ta’zim, Malaysia \\ Correspondence should be addressed to Sergei Alexandrov; sergei_alexandrov@spartak.ru
}

Received 28 December 2013; Revised 24 February 2014; Accepted 24 February 2014; Published 25 March 2014

Academic Editor: Roohollah Jamaati

Copyright (c) 2014 S. Alexandrov and Y. Mustafa. This is an open access article distributed under the Creative Commons Attribution License, which permits unrestricted use, distribution, and reproduction in any medium, provided the original work is properly cited.

\begin{abstract}
The paper presents a theoretical study on the compression of a two-layer strip of strain-hardening rigid-plastic materials between rigid platens. Semianalytical solutions are obtained for stress and velocity fields in each layer. Special attention is devoted to the conditions corresponding to the beginning of cold bond formation between the layers. Depending on input parameters various general deformation patterns are possible. In particular, there exists such a range of process parameters that the soft metal layer yields while the hard metal layer is rigid at the beginning of the process. As the deformation proceeds, yielding also starts in the hard metal layer and the entire strip becomes plastic. This is a typical deformation pattern adopted in describing the process of joining by rolling. However, at a certain range of input parameters plastic deformation of the entire strip begins at the initial instant. Moreover, it is possible that only the hard metal layer yields while the soft metal layer does not. This deformation pattern takes place when the thickness of the soft metal layer is much smaller than that of the hard metal layer.
\end{abstract}

\section{Introduction}

For many years rolling has been recognized as being an important process for joining by forming ([1-7] among many others). Approximate theoretical engineering solutions for this process have been mainly found by the slab method [8-10] or the upper bound method [11-14]. However, none of these methods is really suitable for describing stationary processes with unknown boundaries between layers of different materials. In particular, the upper bound theorem is applicable if and only if the surface configuration including possible interfaces between different materials and the distribution of the local yield stress are known [15]. It is evident that these conditions are not satisfied in multilayer rolling. A possible method to use the upper bound theorem to determine the surface configuration in stationary processes has been proposed in [16]. In order to find a solution by this method, two nested minimization loops are necessary. In the inside loop, the geometry is fixed and the rate of work is minimized with respect to velocities. In the external loop, minimization is carried out with respect to geometry.
This is consistent with the upper bound theorem and is not the same as lumping two loops together. The latter has been however used in solutions for rolling of multilayer sheets. To the best of author's knowledge, no correct upper bound solution is available for such a process. The slab method is also not suitable to describe the process of rolling of multilayer sheets since the shape of bimaterial interfaces should be known in advance to apply this method. Another difficulty with the aforementioned theoretical methods is that their area of applicability, strictly speaking, is restricted to perfectly plastic materials. A typical way to overcome this difficulty is to assume that the yield stress depends on an average value of the equivalent strain (see, e.g., $[10,12]$ ). However, the through thickness distribution of the yield stress is highly nonuniform due to intensive shear deformation near bimaterial interfaces. It is confirmed by highly nonuniform through thickness distributions of hardness (see, e.g., [6]). This effect is revealed in various metal forming process, not only in multilayer rolling (see, e.g., [17-19]). The presence of such a layer of intensive plastic deformation in the vicinity of bimaterial interfaces has a significant effect on the beginning of cold bond formation 
between the layers. For, the position of the bonding point is usually controlled by the increase in the yield stress of the softer layer in the vicinity of the bimaterial interface (see, e.g., [10]). It is however evident from the actual distribution of material properties [6, 17-19] that the replacement of the local yield stress in the vicinity of the bimaterial interface with an average yield stress leads to a significant error. It is worthy of note that since the thickness of the layer of intensive plastic deformation is very small this replacement cannot have a large effect on global parameters such as the rolling power and torque which are typically used to compare theoretical and experimental results. In summary, it is worthwhile to develop an approximate theoretical method for describing multilayer rolling free of the aforementioned drawbacks of the upper bound and slab methods. Actually, a general method satisfying the requirements specified has been proposed in [20]. The method is widely used and shows excellent agreement with experiment for different materials (see, e.g., [21-23]), including rolling of very thin sheets (nanoand mesoscopic scale) as reported in [24]. The method proposed in [20] is used to verify other theories of rolling (see, e.g., [25, 26]). In particular, the solution [26] based on a series expansion shows a better agreement with the Orowan solution as the number of terms in the series expansion increases. Yet, there are very fast methods for solving the final equation obtained in [20] numerically (see, e.g., [27$30]$ ), which is of special importance for design purpose. In order to apply one of the versions of the method [20], it is necessary to have an analytical or semianalytical solution for compression of a multilayer strip between parallel plates. Such solutions are available for strips made of rigid perfectly plastic layers [31] and several combinations of viscoplastic and rigid perfectly plastic layers [32]. However, these material models are not appropriate to describe the change of yield stress due to intensive plastic deformation in the vicinity of the bimaterial interface. Therefore, in the present paper a solution for compression of a two-layer strip of rigid plastic, strain-hardening materials is given. This solution is an extension of the solution proposed in [33] and it constitutes a theoretical basis for using the method [20] to simulate the process of bonding by rolling.

A recent finite element solution for the roll-bonding process has been given in [34]. The solution is based on the numerical procedure developed in [35]. A simplified statement of the boundary value problem has been adopted. In particular, the regime of sticking and the existence of a rigid (or elastic) zone near the neutral point have been ignored. On the other hand, the present exact solution shows that the regime of sticking must occur under certain conditions. Moreover, it follows from the general asymptotic analysis of solution behavior near some friction surfaces for plastic hardening materials carried out in [36] that the sticking zone may occupy the entire friction surface. According to the material model adopted in [34], the yield stress becomes constant if the equivalent strain is high enough. In this case the velocity field becomes singular in the vicinity of some friction surfaces [37]. Presumably, these qualitative features of actual solutions cause difficulties with application of standard finite element methods to boundary value problems in

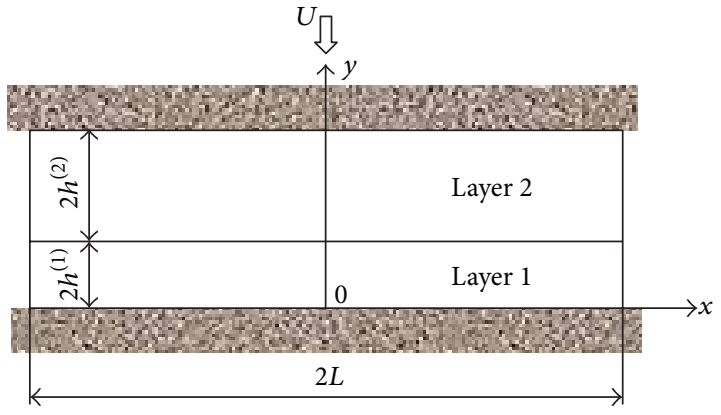

FIGURE 1: Compression of a two-layer strip between rough rigid plates.

metal forming involving surfaces with high friction [38-41]. Therefore, the exact solution given in the present paper can also be used as a benchmark problem for verifying numerical codes developed for simulation of the roll-bonding process. The verification of numerical codes by closed form solutions is a necessary step towards their successful applications to engineering problems $[42,43]$.

\section{Statement of the Problem}

The progressive plane-strain compression of a two-layer strip between parallel, rough plates is considered. The Cartesian coordinate system $(x, y)$ is chosen such that the $x$-axis is parallel to the strip and $y$ measures distance through the strip thickness, as shown in Figure 1.

It will be shown later that the bimaterial interface is a straight line parallel to the $x$-axis. Then the lower surface of the strip is determined by the equation $y=0$, its upper surface by the equation $y=2 h^{(1)}+2 h^{(2)}$, the bimaterial interface by the equation $y=2 h^{(1)}$, and its ends by the equation $x= \pm L$. Here $2 h^{(1)}$ is the current thickness of layer 1 and $2 h^{(2)}$ is the current thickness of layer 2. The initial values of $h^{(1)}$ and $h^{(2)}$ are denoted by $h_{0}^{(1)}$ and $h_{0}^{(2)}$, respectively. The ends $x= \pm L$ are traction-free. The standard stress and velocity boundary conditions are prescribed at the axis of symmetry $x=0$. The other velocity boundary conditions are

$$
u_{y}=-U
$$

for $y=2\left(h^{(1)}+h^{(2)}\right)$, and

$$
u_{y}=0,
$$

for $y=0$. The bimaterial interface moves with the velocity $U_{i}$ whose value should be found from the solution. Thus

$$
u_{y}=-U_{i},
$$

for $y=2 h^{(1)}$. The friction law adopted is associated with the material model and, therefore, the corresponding boundary conditions at $y=0, y=2 h^{(1)}$, and $y=2\left(h^{(1)}+h^{(2)}\right)$ will be formulated later. The boundary value problem is symmetric relative to the $y$-axis. Therefore, it is sufficient to find the solution in the range $x \geq 0$. The standard constitutive equations of 
rigid/plastic, strain-hardening material are adopted for each layer. In particular, the yield criteria are

$$
\begin{aligned}
& \left(\sigma_{x x}-\sigma_{y y}\right)^{2}+4 \sigma_{x y}^{2}=4\left[k_{0}^{(1)} \Phi_{1}\left(\varepsilon_{\mathrm{eq}}\right)\right]^{2}, \\
& \left(\sigma_{x x}-\sigma_{y y}\right)^{2}+4 \sigma_{x y}^{2}=4\left[k_{0}^{(2)} \Phi_{2}\left(\varepsilon_{\mathrm{eq}}\right)\right]^{2},
\end{aligned}
$$

for layers 1 and 2, respectively. Here $\sigma_{x x}, \sigma_{y y}$, and $\sigma_{x y}$ are the stress components in the Cartesian coordinates, $k_{0}^{(1)}$ is the initial shear yield stress in layer $1, k_{0}^{(2)}$ is the initial shear yield stress in layer 2 , and $\Phi_{1}\left(\varepsilon_{\mathrm{eq}}\right)$ and $\Phi_{2}\left(\varepsilon_{\mathrm{eq}}\right)$ are arbitrary monotonically increasing functions of the equivalent strain, $\varepsilon_{\text {eq }}$, subject to $\Phi_{1}(0)=\Phi_{2}(0)=1$. The equivalent strain is defined as

$$
\frac{d \varepsilon_{\mathrm{eq}}}{d t}=\xi_{\mathrm{eq}}
$$

where $d / d t$ stands for the convected derivative and $\xi_{\mathrm{eq}}$ is the equivalent strain rate defined by

$$
\xi_{\mathrm{eq}}=\sqrt{\frac{\left(\xi_{x x}-\xi_{y y}\right)^{2}}{4}+\xi_{x y}^{2}} .
$$

Here $\xi_{x x}, \xi_{y y}$, and $\xi_{x y}$ are the components of the strain rate tensor in the Cartesian coordinate system. The associated flow rule gives

$$
\begin{gathered}
\xi_{x x}=\frac{\xi_{\mathrm{eq}}\left(\sigma_{x x}-\sigma_{y y}\right)}{2 k_{0}^{(1)} \Phi_{1}\left(\varepsilon_{\mathrm{eq}}\right)}, \\
\xi_{y y}=\frac{\xi_{\mathrm{eq}}\left(\sigma_{y y}-\sigma_{x x}\right)}{2 k_{0}^{(1)} \Phi_{1}\left(\varepsilon_{\mathrm{eq}}\right)}, \\
\xi_{x y}=\frac{\xi_{\mathrm{eq}} \sigma_{x y}}{k_{0}^{(1)} \Phi_{1}\left(\varepsilon_{\mathrm{eq}}\right)},
\end{gathered}
$$

in layer 1 , and

$$
\begin{gathered}
\xi_{x x}=\frac{\xi_{\mathrm{eq}}\left(\sigma_{x x}-\sigma_{y y}\right)}{2 k_{0}^{(2)} \Phi_{2}\left(\varepsilon_{\mathrm{eq}}\right)}, \\
\xi_{y y}=\frac{\xi_{\mathrm{eq}}\left(\sigma_{y y}-\sigma_{x x}\right)}{2 k_{0}^{(2)} \Phi_{2}\left(\varepsilon_{\mathrm{eq}}\right)}, \\
\xi_{x y}=\frac{\xi_{\mathrm{eq}} \sigma_{x y}}{k_{0}^{(2)} \Phi_{2}\left(\varepsilon_{\mathrm{eq}}\right)},
\end{gathered}
$$

in layer 2. A conventional friction law in metal forming is to take the friction stress to be a constant fraction of the local yield shear stress. In this case the frictional boundary conditions are

$$
T_{2} \leq m_{2} k_{0}^{(2)} \Phi_{2}\left(\varepsilon_{\mathrm{eq}}\right),
$$

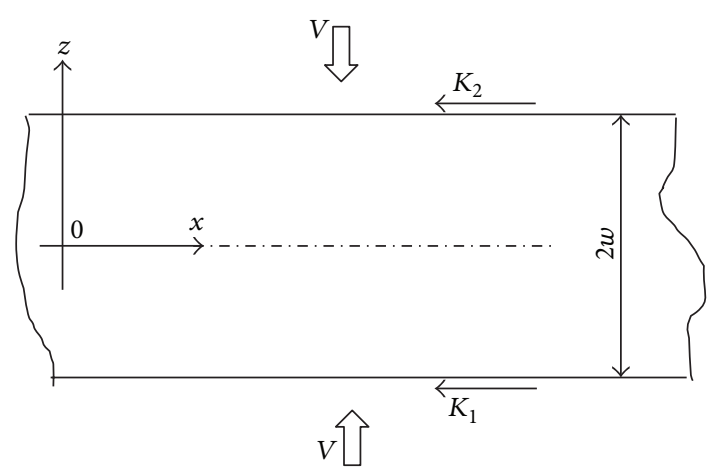

FIGURE 2: Compression of a single layer between rough rigid plates.

for $y=2\left(h^{(1)}+h^{(2)}\right)$,

$$
T_{1} \leq m_{1} k_{0}^{(1)} \Phi_{1}\left(\varepsilon_{\mathrm{eq}}\right),
$$

for $y=0$, and

$$
\left|T_{i}\right| \leq m_{i} \min \left\{k_{0}^{(1)} \Phi_{1}\left(\varepsilon_{\mathrm{eq}}\right), k_{0}^{(2)} \Phi_{2}\left(\varepsilon_{\mathrm{eq}}\right)\right\},
$$

for $y=2 h^{(1)}$. With no loss of generality it is possible to assume that $k_{0}^{(1)}>k_{0}^{(2)}$. Then,

$$
k_{0}^{(1)}=\alpha k_{0}^{(2)}, \quad \alpha>1 .
$$

The system of (4a), (4b), (7), and (8) is supplemented with the equilibrium equations.

\section{An Exact Instantaneous Solution for One Layer}

An exact solution of (4a) and (7) (or (4b) and (8)) supplemented with the equilibrium equations has been derived in [33]. In order to use this solution, it is convenient to introduce new notations. A schematic diagram of the layer under consideration and Cartesian coordinates $(x, z)$ are shown in Figure 2. The plates are now moving toward each other with speed $V$. The magnitude of the friction stress is equal to $K_{1}$ at $z=-w$ and $K_{2}$ at $z=w$. These stresses are directed as shown in Figure 2. The boundary conditions at $x= \pm L$ and $x=0$ (Figure 1) are ignored. The constitutive equations (4a) and (7) (or (4b) and (8)) are replaced with

$$
\begin{gathered}
\left(\sigma_{x x}-\sigma_{y y}\right)^{2}+4 \sigma_{x y}^{2}=4\left[k_{0} \Phi\left(\varepsilon_{\mathrm{eq}}\right)\right]^{2}, \\
\xi_{x x}=\frac{\xi_{\mathrm{eq}}\left(\sigma_{x x}-\sigma_{y y}\right)}{2 k_{0} \Phi\left(\varepsilon_{\mathrm{eq}}\right)}, \\
\xi_{y y}=\frac{\xi_{\mathrm{eq}}\left(\sigma_{y y}-\sigma_{x x}\right)}{2 k_{0} \Phi\left(\varepsilon_{\mathrm{eq}}\right)} \\
\xi_{x y}=\frac{\xi_{\mathrm{eq}} \sigma_{x y}}{k_{0} \Phi\left(\varepsilon_{\mathrm{eq}}\right)} .
\end{gathered}
$$


The solution to the equilibrium equations and (13) proposed in $[33]$ is

$$
\begin{aligned}
\sigma_{x y} & =-\frac{\bar{K} z}{w}+\Delta, \quad \sigma_{y y}=\frac{\bar{K} x}{w}+c, \\
\sigma_{x x} & =\frac{\bar{K} x}{w}+c+2 \sqrt{k_{0}^{2} \Phi^{2}\left(\varepsilon_{\mathrm{eq}}\right)-\sigma_{x y}^{2}} \\
u_{x} & =\frac{V x}{w}-\frac{2 V}{w} \int_{z_{*}}^{z} \cot 2 \phi d \beta+C, \\
u_{z} & =-\frac{V z}{w} .
\end{aligned}
$$

Here $u_{x}$ and $u_{z}$ are the velocity components in the Cartesian coordinates, $c$ and $C$ are constants of integration, $z_{*}$ is an arbitrary constant introduced for further convenience, and $\beta$ is a dummy variable of integration. $\phi$ depends on $z$ and does not depend on $x$. Also,

$$
\begin{gathered}
\bar{K}=\frac{1}{2}\left(K_{1}+K_{2}\right), \quad \Delta=\frac{1}{2}\left(K_{1}-K_{2}\right), \\
\cos 2 \phi=-\frac{\bar{K} z}{w k_{0} \Phi\left(\varepsilon_{\mathrm{eq}}\right)}+\frac{\Delta}{k_{0} \Phi\left(\varepsilon_{\mathrm{eq}}\right)} .
\end{gathered}
$$

The equation for $\phi$ is compatible with the requirement that $\phi$ is independent of $x$ if $\varepsilon_{\mathrm{eq}}$ is independent of $x$. The latter is satisfied since it follows from (6) and (15a), (15b) that

$$
\xi_{\mathrm{eq}}=\frac{V}{w}|\operatorname{cosec} 2 \phi|
$$

and, thus, the right hand side of (5) is independent of $x$. Equation (15b) demands that all lines parallel to the $x$-axis at the initial instant remain parallel to this axis in the progressive compression.

\section{An Instantaneous Solution for the Strip of Two Plastic Layers}

The solution described in the previous section can be adopted to find a solution for the boundary value problem formulated in Section 2. First it is assumed that both layers are plastic. Then the solution given in Section 3 is valid in each of these layers. The solution $((15 \mathrm{a}),(15 \mathrm{~b}))$ for $u_{x}$ shows that the regime of sliding occurs at the frictional interfaces $y=0$ and $y=$ $2\left(h^{(1)}+h^{(2)}\right)$ (Figure 1). Therefore, the boundary conditions (9) and (10) become

$$
T_{2}=m_{2} k_{0}^{(2)} \Phi_{2}\left(\varepsilon_{\mathrm{eq}}\right),
$$

for $y=2\left(h^{(1)}+h^{(2)}\right)$, and

$$
T_{1}=m_{1} k_{0}^{(1)} \Phi_{1}\left(\varepsilon_{\mathrm{eq}}\right),
$$

for $y=0$, respectively. The velocity boundary conditions (1) to (3) can be transformed to the velocity boundary conditions used in the solution given in Section 3 by superimposing

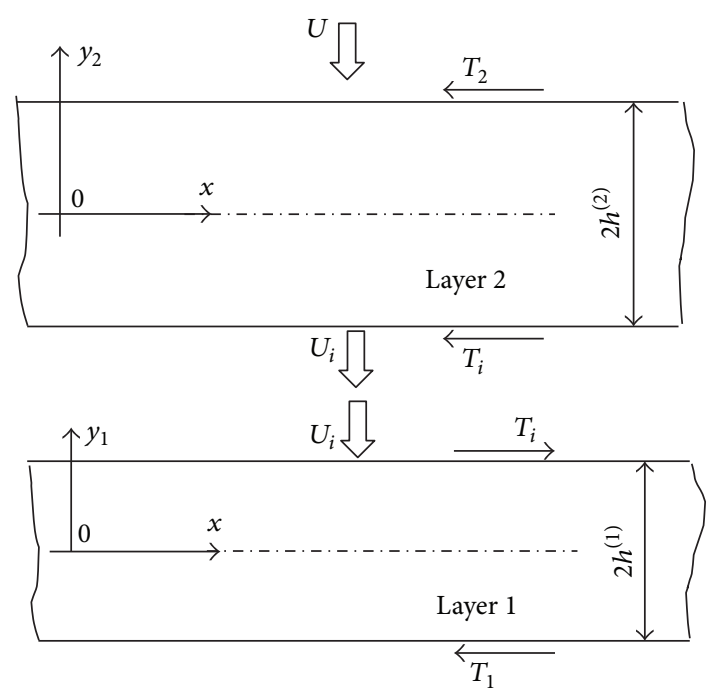

FIGURE 3: Boundary conditions and coordinate systems for each layer.

a rigid body translation on the velocity field in each layer. In particular, it is necessary to put

$$
\begin{aligned}
& V=\frac{U_{i}}{2}, \\
& V=\frac{U-U_{i}}{2},
\end{aligned}
$$

for layers 1 and 2, respectively. It is also convenient to introduce new coordinates $\left(x, y_{1}\right)$ and $\left(x, y_{2}\right)$, as shown in Figure 3.

To apply the solution (14) and ((15a), (15b)) to layer 2 , it is necessary to put (Figures 2 and 3) $k_{0}=k_{0}^{(2)}, \Phi\left(\varepsilon_{\mathrm{eq}}\right)=\Phi_{2}\left(\varepsilon_{\mathrm{eq}}\right)$, $c=c_{2}, C=C_{2}, K_{2}=T_{2}, K_{1}=T_{i}, z=y_{2}$, and $w=h^{(2)}$. Then, from (14) and (16),

$$
\begin{aligned}
\sigma_{x y} & =-\frac{\left(T_{2}+T_{i}\right) y_{2}}{2 h^{(2)}}+\frac{T_{i}-T_{2}}{2}, \\
\sigma_{y y} & =\frac{\left(T_{2}+T_{i}\right) x}{2 h^{(2)}}+c_{2}, \\
\sigma_{x x} & =\frac{\left(T_{2}+T_{i}\right) x}{h^{(2)}}+c_{2}+2 \sqrt{\left[k_{0}^{(2)} \Phi_{2}\left(\varepsilon_{\mathrm{eq}}\right)\right]^{2}-\sigma_{x y}^{2} .}
\end{aligned}
$$

Assuming $z_{*}=-h^{(2)}$ and eliminating $V$ by means of (20b) the solution $((15 a),(15 b))$ for the velocities is transformed to

$$
\begin{gathered}
u_{x}=\frac{x\left(U-U_{i}\right)}{2 h^{(2)}}-\frac{\left(U-U_{i}\right)}{h^{(2)}} \int_{-h^{(2)}}^{y_{2}} \cot 2 \phi d \beta+C_{2}, \\
u_{y}=-\frac{y_{2}\left(U-U_{i}\right)}{2 h^{(2)}} .
\end{gathered}
$$

To apply the solution (14) and ((15a), (15b)) to layer 1 , it is necessary to put $k_{0}=k_{0}^{(1)}, \Phi\left(\varepsilon_{\mathrm{eq}}\right)=\Phi_{1}\left(\varepsilon_{\mathrm{eq}}\right), c=c_{1}, C=C_{1}$, 
$K_{2}=-T_{i}, K_{1}=T_{1}, z=y_{1}$, and $w=h^{(1)}$. Then, from (14) and (16),

$$
\begin{aligned}
\sigma_{x y} & =-\frac{\left(T_{1}-T_{i}\right) y_{1}}{2 h^{(1)}}+\frac{T_{1}+T_{i}}{2}, \\
\sigma_{y y} & =\frac{\left(T_{1}-T_{i}\right) x}{2 h^{(1)}}+c_{1}, \\
\sigma_{x x} & =\frac{\left(T_{1}-T_{i}\right) x}{2 h^{(1)}}+c_{1}+2 \sqrt{\left[k_{0}^{(1)} \Phi_{1}\left(\varepsilon_{\mathrm{eq}}\right)\right]^{2}-\sigma_{x y}^{2}} .
\end{aligned}
$$

Assuming $z_{*}=h^{(1)}$ and eliminating $V$ by means of (20a) the solution $((15 \mathrm{a}),(15 \mathrm{~b}))$ for the velocities is transformed to

$$
u_{x}=\frac{U_{i} x}{2 h^{(1)}}-\frac{U_{i}}{h^{(1)}} \int_{h^{(1)}}^{y_{1}} \cot 2 \phi d \beta+C_{1}, \quad u_{y}=-\frac{U_{i} y_{1}}{2 h^{(1)}} .
$$

The stress $\sigma_{y y}$ must be continuous across the bimaterial interface. Therefore, it follows from (21) and (23) that

$$
c_{1}=c_{2}
$$

and $\left(T_{2}+T_{i}\right) h^{(1)}=\left(T_{1}-T_{i}\right) h^{(2)}$. Solving this equation for $T_{i}$ yields

$$
T_{i}=\frac{\left(s T_{1}-T_{2}\right)}{(1+s)},
$$

where $s=h^{(2)} / h^{(1)}$. The solution found exists if and only if (26) is compatible with (11). In general, there exist two critical values of $s, s_{c 1}$, and $s_{c 2}$ such that (11) is not satisfied in the ranges $s>s_{c 1}$ and $s<s_{c 2}$, respectively. In these cases one of the layers is rigid and the regime of sliding occurs at the bimaterial interface. The values of $s_{c 1}$ and $s_{c 2}$ can be found from (26) assuming that

$$
\begin{gathered}
T_{i}=m_{i} \min \left\{k_{0}^{(1)} \Phi_{1}\left(\varepsilon_{\mathrm{eq}}\right), k_{0}^{(2)} \Phi_{2}\left(\varepsilon_{\mathrm{eq}}\right)\right\}, \\
T_{i}=-m_{i} \min \left\{k_{0}^{(1)} \Phi_{1}\left(\varepsilon_{\mathrm{eq}}\right), k_{0}^{(2)} \Phi_{2}\left(\varepsilon_{\mathrm{eq}}\right)\right\},
\end{gathered}
$$

respectively. In the range $s_{c 2}<s<s_{c 1}$ the regime of sticking occurs at the bimaterial interface. Therefore, the velocity component $u_{x}$ must be continuous across this interface, where $y_{2}=-h^{(2)}$ and $y_{1}=h^{(1)}$. Using (22) and (24) it is possible to find that this condition is satisfied if

$$
C_{1}=C_{2}, \quad U_{i}=\frac{U}{s+1}
$$

\section{An Instantaneous Solution for the Strip of One Plastic Layer}

Layer 1 is rigid when $s>s_{c 1}$. The regime of sliding occurs at the bimaterial interface. Therefore, (11) is transformed to

$$
T_{i}=m_{i} \min \left\{k_{0}^{(1)} \Phi_{1}\left(\varepsilon_{\mathrm{eq}}\right), k_{0}^{(2)} \Phi_{2}\left(\varepsilon_{\mathrm{eq}}\right)\right\} .
$$

Since layer 1 is rigid, then $U_{i}=0$. The solution given by (14) and ((15a), (15b)) in which $K_{1}$ should be replaced with $T_{i}, K_{2}$ with $T_{2}, w$ with $h^{(2)}, k_{0}$ with $k_{0}^{(2)}, \Phi\left(\varepsilon_{\text {eq }}\right)$ with $\Phi_{2}\left(\varepsilon_{\text {eq }}\right), z$ with $y_{2}$, and $V$ with $U / 2$ is valid. It is convenient to take $z_{*}=-h^{(2)}$. Then, using (16), (19), and (29),

$$
\begin{aligned}
\sigma_{x y} & =-\frac{\left(T_{2}+T_{i}\right) y_{2}}{2 h^{(2)}}+\frac{T_{i}-T_{2}}{2}, \quad \sigma_{y y}=\frac{\left(T_{2}+T_{i}\right) x}{2 h^{(2)}}+c, \\
\sigma_{x x} & =\frac{\left(T_{2}+T_{i}\right) x}{2 h^{(2)}}+c+2 \sqrt{\left[k_{0}^{(2)} \Phi_{2}\left(\varepsilon_{\mathrm{eq}}\right)\right]^{2}-\sigma_{x y}^{2}} \\
u_{x} & =\frac{U x}{2 h^{(2)}}-\frac{U}{h^{(2)}} \int_{-h^{(2)}}^{y_{2}} \cot 2 \phi d \beta+C, \quad u_{y}=-\frac{U y_{2}}{2 h^{(2)}} .
\end{aligned}
$$

Layer 2 is rigid when $s<s_{c 2}$. The regime of sliding occurs at the bimaterial interface. Therefore, (29) is valid. Since layer 2 is rigid, then $U_{i}=U$. The solution given by (14) and ((15a), (15b)) in which $K_{1}$ should be replaced with $T_{1}, K_{2}$ with $-T_{i}$, $w$ with $h^{(1)}, k_{0}$ with $k_{0}^{(1)}, \Phi\left(\varepsilon_{\mathrm{eq}}\right)$ with $\Phi_{1}\left(\varepsilon_{\mathrm{eq}}\right), z$ with $y_{1}$, and $V$ with $U / 2$ is valid. It is convenient to take $z_{*}=h^{(1)}$. Then, using (16), (19), and (29),

$$
\begin{aligned}
\sigma_{x y} & =-\frac{\left(T_{1}-T_{i}\right) y_{1}}{2 h^{(1)}}+\frac{T_{1}+T_{i}}{2}, \quad \sigma_{y y}=\frac{\left(T_{1}-T_{i}\right) x}{2 h^{(1)}}+c, \\
\sigma_{x x} & =\frac{\left(T_{1}-T_{i}\right) x}{2 h^{(1)}}+c+2 \sqrt{\left[k_{0}^{(1)} \Phi_{1}\left(\varepsilon_{\mathrm{eq}}\right)\right]^{2}-\sigma_{x y}^{2}}, \\
u_{x} & =\frac{U x}{2 h^{(1)}}-\frac{U}{h^{(1)}} \int_{h^{(1)}}^{y_{1}} \cot 2 \phi d \beta+C, \quad u_{y}=-\frac{U y_{1}}{2 h^{(1)}} .
\end{aligned}
$$

\section{Cold Bond Formation in Continued Compression of Two-Layer Strips}

Replacing $T_{i}$ in (26) with (27a) and then with (27b) at $\varepsilon_{\mathrm{eq}}=0$ and using (12), (18), and (19) give the following equations for $s_{c 1}$ and $s_{c 2}$ at the initial instant:

$$
\begin{aligned}
& s_{c 1}=\frac{m_{i}+m_{2}}{\alpha m_{1}-m_{i}}, \\
& s_{c 2}=\frac{m_{2}-m_{i}}{\alpha m_{1}+m_{i}} .
\end{aligned}
$$

Each of these expressions involves four independent input parameters. Therefore, it is impossible to provide a clear geometric illustration of this solution in its generality. In order to give some insights into qualitative features of the solution, it is assumed that $m_{1}=m_{2}=0.7$ and $\alpha=3$. Then, both $s_{c 1}$ and $s_{c 2}$ solely depend on $m_{i}$. The corresponding curves are depicted in Figure 4 , where $s_{0}=h_{0}^{(2)} / h_{0}^{(1)}$. The domain between the curves corresponds to the regime of sticking at the bimaterial interface at the initial instant. The curves intersect at $m_{i}=0$. It follows from $((32 a),(32 b))$ that in this special case the regime of sticking at the initial instant can only occur if $h_{0}^{(2)} / h_{0}^{(1)}=m_{2} /\left(\alpha m_{1}\right)$. The lower curve intersects 


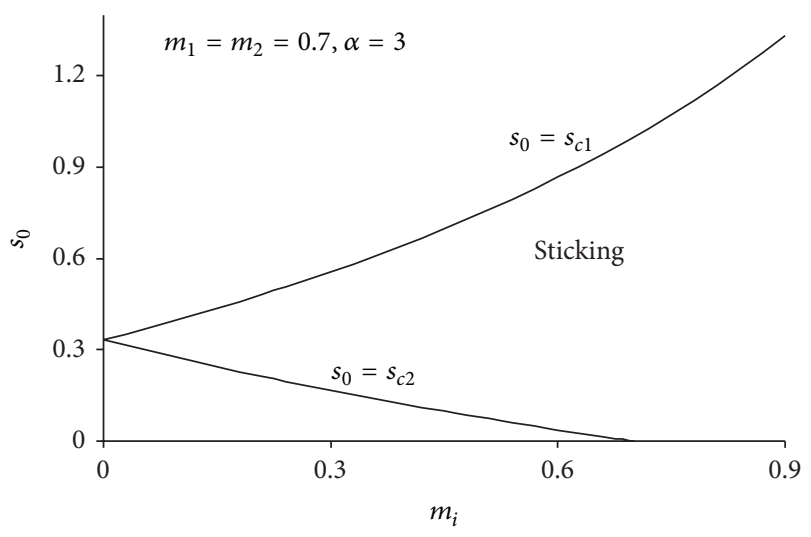

FIGURE 4: Domain of sticking at the initial instant.

the horizontal axis at $m_{i}=m_{2}$. It is evident from (32b). The physical meaning of this mathematical feature of the solution is that the soft layer is plastic at $m_{i} \geq m_{2}$ even if its thickness is vanishing. On the other hand, it follows from (32a) that $s_{c 1} \rightarrow \infty$ as $m_{i} \rightarrow \alpha m_{1}$. It is evident that it is possible if and only if $\alpha m_{1}<1$. Therefore, this feature of the solution is not revealed in Figure 4. Its physical meaning is that the regime of sticking at the bimaterial interface occurs at any large value of the ratio $h_{0}^{(2)} / h_{0}^{(1)}$. In what follows, it is assumed that the set of input parameters is such that the hard layer is rigid and the soft layer is plastic at the initial instant. This is a typical situation in rolling of two-layer strips [6]. It will be also assumed that the materials are linear strain hardening. Thus

$$
\begin{aligned}
& \Phi_{1}\left(\varepsilon_{\mathrm{eq}}\right)=1+H_{1} \varepsilon_{\mathrm{eq}}, \\
& \Phi_{2}\left(\varepsilon_{\mathrm{eq}}\right)=1+H_{2} \varepsilon_{\mathrm{eq}}
\end{aligned}
$$

where $H_{1}$ and $H_{2}$ are constant hardening moduli.

Since the hard layer is rigid during an initial stage of the process of compression, $h^{(1)}=h_{0}^{(1)}$ and $\varepsilon_{\mathrm{eq}}=0$ in this layer. The solution (30) is valid in the soft layer. In order to use (26), it is necessary to find the dependence of $T_{i}$ and $T_{2}$ on $\lambda^{(2)}=$ $\ln \left(h^{(2)} / h_{0}^{(2)}\right)$. Replacing $K_{1}$ with $T_{i}, K_{2}$ with $T_{2}, w$ with $h^{(2)}, k_{0}$ with $k_{0}^{(2)}, z$ with $y_{2}, V$ with $U / 2$, and $\Phi\left(\varepsilon_{\text {eq }}\right)$ with (33b) in (16) and (17) yields

$$
\begin{gathered}
\cos 2 \phi=-\frac{\left[m_{2}\left(1+H_{2} \varepsilon_{2}\right)+m_{i}\left(1+H_{2} \varepsilon_{i}\right)\right] y_{2}}{2 h^{(2)}\left(1+H_{2} \varepsilon_{\mathrm{eq}}\right)} \\
+\frac{m_{i}\left(1+H_{2} \varepsilon_{i}\right)-m_{2}\left(1+H_{2} \varepsilon_{2}\right)}{2\left(1+H_{2} \varepsilon_{\mathrm{eq}}\right)}, \\
\xi_{\mathrm{eq}}=\frac{U}{2 h^{(2)}}|\operatorname{cosec} 2 \phi| .
\end{gathered}
$$

Here $\varepsilon_{2}$ is the value of $\varepsilon_{\mathrm{eq}}$ at $y_{2}=h^{(2)}$ and $\varepsilon_{i}$ is the value of $\varepsilon_{\text {eq }}$ at $y_{2}=-h^{(2)}$. Equations (19) and (29) have been used to eliminate $T_{1}$ and $T_{i}$. Note that $d h^{(2)} / d t=-U / 2$. Therefore, it follows from (5) and (34b) that

$$
\frac{d \varepsilon_{\mathrm{eq}}}{d \lambda^{(2)}}=-|\operatorname{cosec} 2 \phi| \text {. }
$$

It follows from (34a) that

$$
\cos 2 \phi=-m_{2}, \quad \cos 2 \phi=m_{i},
$$

at $y_{2}=h^{(2)}$ and $y_{2}=-h^{(2)}$, respectively. Substituting (36) into (35) and integrating with the use of the initial condition $\varepsilon_{\text {eq }}=0$ at $\lambda^{(2)}=0$ give

$$
\varepsilon_{2}=-\frac{\lambda^{(2)}}{\sqrt{1-m_{2}^{2}}}, \quad \varepsilon_{i}=-\frac{\lambda^{(2)}}{\sqrt{1-m_{i}^{2}}}
$$

Substituting (37) into (33a), (33b) and the resulting equations into (19) and (29) leads to

$$
\begin{aligned}
& T_{2}=m_{2} k_{0}^{(2)}\left(1-H_{2} \frac{\lambda^{(2)}}{\sqrt{1-m_{2}^{2}}}\right), \\
& T_{i}=m_{i} k_{0}^{(2)}\left(1-H_{2} \frac{\lambda^{(2)}}{\sqrt{1-m_{i}^{2}}}\right) .
\end{aligned}
$$

Combining (26) and (38) gives the following equation for the value of $\lambda^{(2)}=\lambda_{c}$ corresponding to the beginning of cold bond formation between the layers:

$$
\begin{aligned}
& m_{i}\left(1-H_{2} \frac{\lambda_{c}}{\sqrt{1-m_{i}^{2}}}\right)\left(1+s_{0} \exp \lambda_{c}\right) \\
& =\alpha m_{1} s_{0} \exp \lambda_{c}-m_{2}\left(1-H_{2} \frac{\lambda_{c}}{\sqrt{1-m_{2}^{2}}}\right) .
\end{aligned}
$$

It has been taken into account here that $T_{1}=m_{1} k_{0}^{(1)}$ at this instant. At $\lambda^{(2)}<\lambda_{c}$ the solution given in Section 4 should be used. In particular, it follows from (22) and (24) that

$$
\frac{d h^{(2)}}{d t}=-\frac{U-U_{i}}{2}, \quad \frac{d h^{(1)}}{d t}=-\frac{U_{i}}{2} .
$$

Combining these equations and (28) gives

$$
\frac{d h^{(2)}}{d h^{(1)}}=\frac{U}{U_{i}}-1=s=\frac{h^{(2)}}{h^{(1)}} .
$$

Integrating this equation with the use of the initial condition $h^{(1)}=h_{0}^{(1)}$ at $h^{(2)}=h_{0}^{(2)} \exp \lambda_{c}$ yields

$$
\frac{h^{(2)}}{h^{(1)}}=\frac{h_{0}^{(2)}}{h_{0}^{(1)}} \exp \lambda_{c} \text {. }
$$




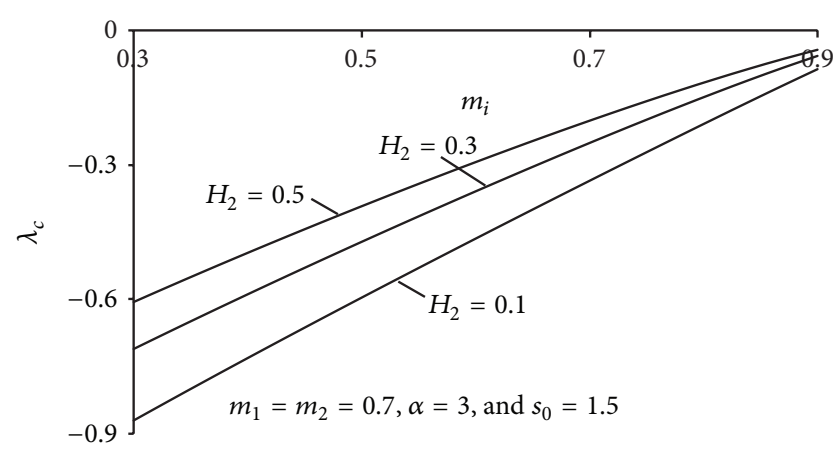

Figure 5: Variation of $\lambda_{c}$ with $m_{i}$ at different values of $H_{2}$ (other input parameters are fixed).

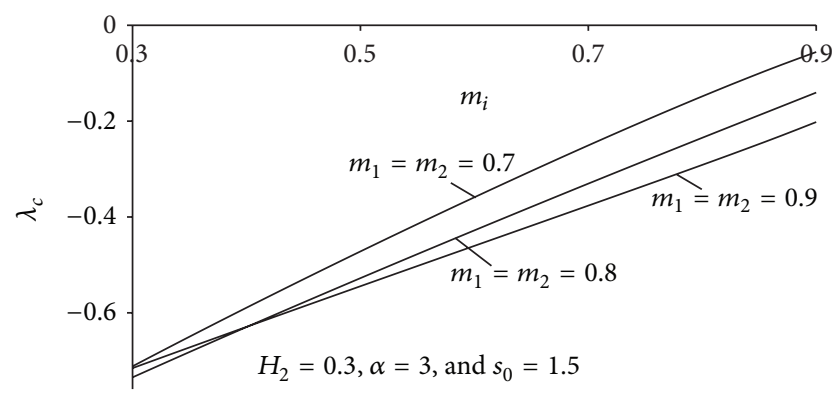

FIGURE 6: Variation of $\lambda_{c}$ with $m_{i}$ at different values of $m_{1}=m_{2}$ (other input parameters are fixed).

The total thickness of the strip at the end of the process is given by

$$
h_{f}=2\left(h^{(1)}+h^{(2)}\right) .
$$

Equations (42) and (43) can be used to find the thickness of the individual layers at the end of the process as

$$
\begin{aligned}
& h_{f}^{(2)}=\frac{h_{f} \exp \lambda_{c}}{2\left(h_{0}^{(1)} / h_{0}^{(2)}+\exp \lambda_{c}\right)}, \\
& h_{f}^{(1)}=\frac{h_{f}}{2\left[1+\left(h_{0}^{(2)} / h_{0}^{(1)}\right) \exp \lambda_{c}\right]} .
\end{aligned}
$$

It is seen from this equation that the parameter $\lambda_{c}$ controls the final configuration of the product. In order to illustrate the dependence of $\lambda_{c}$ on input parameters, (39) has been solved numerically. The variation of $\lambda_{c}$ with $m_{i}$ is depicted in Figures 5 to 7 . In all cases the magnitude of $\lambda_{c}$ decreases with $m_{i}$. Figure 5 illustrates the effect of the hardening modulus $\mathrm{H}_{2}$ on $\lambda_{c}$. It is seen from this figure that the magnitude of $\lambda_{c}$ decreases as $\mathrm{H}_{2}$ increases. Figure 6 shows that the dependence of the magnitude of $\lambda_{c}$ on $m_{1}$ and $m_{2}$ when $m_{1}=$ $m_{2}$ is not monotonic at small $m_{i}$. However, when $m_{i}$ is large enough the magnitude of $\lambda_{c}$ decreases with these friction factors. Finally, it is seen from Figure 7 that the magnitude of $\lambda_{c}$ decreases with the initial ratio $s_{0}=h_{0}^{(2)} / h_{0}^{(1)}$.

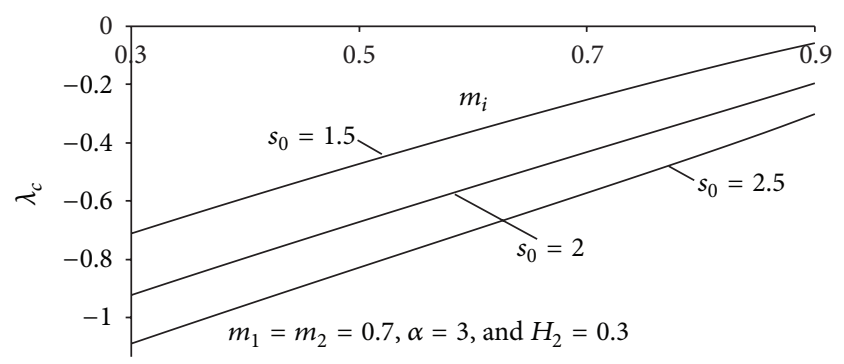

FIGURE 7: Variation of $\lambda_{c}$ with $m_{i}$ at different values of $s_{0}$ (other input parameters are fixed).

\section{Conclusions}

A semianalytical solution for the compression of a twolayer strip of strain-hardening materials has been given. Numerical treatment has been reduced to solving a transcendental equation (39) and evaluating ordinary integrals. The solution to the transcendental equation predicts the thickness of the soft layer corresponding to the beginning of cold bold formation between the layers. Then, the final thickness of each layer is determined from (44) if the final thickness of the strip is given. Evaluating ordinary integrals is required to obtain detailed information on the through thickness distribution of stresses, strains, and velocities. The solution found determines the conditions of the transition between the regimes of sticking and sliding. These conditions are of special importance for the process of bonding.

The process of bonding by rolling can be simulated using the approach proposed in [20]. In this case, $s_{0}$ is determined by the thickness of each layer at the entrance to the roll gap and $h_{f}$ introduced in (43) by the thickness of the strip at the exit. It is worthy of note that powerful numerical methods have been already developed to simulate the rolling process using this approach (see, e.g., [28]) and the present solution can be directly incorporated in simulations of the roll-bonding process by means of these numerical methods.

The solution found reduces to the rigid perfectly plastic solution given in [31] if $H_{1}=H_{2}=0$ in (33a), (33b). The latter is in qualitative agreement with the experimental data reported in [44]. The solution can also serve as a benchmark problem for verifying numerical codes since accurate closed form solutions are necessary for this purpose [42].

\section{Conflict of Interests}

The authors declare that there is no conflict of interests regarding the publication of this paper.

\section{Acknowledgment}

The research described in this paper was supported by Grants RFBR-13-08-00969 and NSH-1275.2014.1 (Russia). 


\section{References}

[1] J. J. Moore, D. V. Wilson, and W. T. Roberts, "Fabrication of formable metal-metal composites," Materials Science and Engineering, vol. 48, no. 1, pp. 113-121, 1981.

[2] N. Bay, C. Clemensen, O. Juelstorp, and T. Wanheim, "Bond strength in cold roll bonding," CIRP Annals-Manufacturing Technology, vol. 34, no. 1, pp. 221-224, 1985.

[3] D. Pan, K. Gao, and J. Yu, "Cold roll bonding of bimetallic sheets and strips," Materials Science and Technology, vol. 5, no. 9, pp. 934-939, 1989.

[4] F. Carreño, J. Chao, M. Pozuelo, and O. A. Ruano, "Microstructure and fracture properties of an ultrahigh carbon steel-mild steel laminated composite," Scripta Materialia, vol. 48, no. 8, pp. 1135-1140, 2003.

[5] H. D. Manesh and A. K. Taheri, "Study of mechanisms of cold roll welding of aluminium alloy to steel strip," Materials Science and Technology, vol. 20, no. 8, pp. 1064-1068, 2004.

[6] X. Li, G. Zu, M. Ding, Y. Mu, and P. Wang, "Interfacial microstructure and mechanical properties of $\mathrm{Cu} / \mathrm{Al}$ clad sheet fabricated by asymmetrical roll bonding and annealing," Materials Science and Engineering A, vol. 529, no. 1, pp. 485-491, 2011.

[7] X. Li, G. Zu, and P. Wang, "Interface strengthening of laminated composite produced by asymmetrical roll bonding," Materials Science and Engineering A, vol. 562, pp. 96-100, 2013.

[8] A. A. Afonja and D. H. Sansome, "A theoretical analysis of the sandwich rolling process," International Journal of Mechanical Sciences, vol. 15, no. 1, pp. 1-14, 1973.

[9] S. Lee and D. N. Lee, "Slab analysis of roll bonding of silver clad phosphor bronze sheets," Materials Science and Technology, vol. 7, no. 11, pp. 1042-1050, 1991.

[10] G. P. Chaudhari and V. Acoff, "Cold roll bonding of multilayered bi-metal laminate composites," Composites Science and Technology, vol. 69, no. 10, pp. 1667-1675, 2009.

[11] Y.-M. Hwang, H.-H. Hsu, and H.-J. Lee, "Analysis of sandwich sheet rolling by stream function method," International Journal of Mechanical Sciences, vol. 37, no. 3, pp. 297-315, 1995.

[12] Y.-M. Hwang, H.-H. Hsu, and Y.-L. Hwang, "Analytical and experimental study on bonding behavior at the roll gap during complex rolling of sandwich sheets," International Journal of Mechanical Sciences, vol. 42, no. 12, pp. 2417-2437, 2000.

[13] H. Pishbin, M. H. Parsa, and A. Dastvareh, "An analytical modified model of clad sheet bonding by cold rolling using upper bond theorem," Journal of Materials Engineering and Performance, vol. 19, no. 7, pp. 936-941, 2010.

[14] H. Maleki, S. Bagherzadeh, B. Mollaei-Dariani, and K. Abrinia, "Analysis of bonding behavior and critical reduction of twolayer strips in clad cold rolling process," Journal of Engineering Materials and Performance, vol. 22, no. 4, pp. 917-925, 2013.

[15] R. Hill, "A general method of analysis for metal-working processes," Journal of the Mechanics and Physics of Solids, vol. 11, no. 5, pp. 305-326, 1963.

[16] A. Azarkhin and O. Richmond, "A model of ploughing by a pyramidal indenter-upper bound method for stress-free surfaces," Wear, vol. 157, no. 2, pp. 409-418, 1992.

[17] S. P. Moylan, S. Kompella, S. Chandrasekar, and T. N. Farris, "A new approach for studying mechanical properties of thin surface layers affected by manufacturing processes," Transactions of the ASME Journal of Manufacturing Science and Engineering, vol. 125, no. 2, pp. 310-315, 2003.
[18] T. A. Trunina and E. A. Kokovikhin, "Formation of a finely dispersed structure in steel surface layers under combined processing using hydraulic pressing," Journal of Machinery Manufacture and Reliability, vol. 37, no. 2, pp. 160-162, 2008.

[19] S. Aleksandrov, D. Grabko, and O. Shikimaka, "The determination of the thickness of a layer of intensive deformations in the vicinity of the friction surface in metal forming processes," Journal of Machinery Manufacture and Reliability, vol. 38, no. 3, pp. 277-282, 2009.

[20] E. Orowan, "The calculation of roll pressure in hot and cold flat rolling," Proceedings of the Institution of Mechanical Engineers, vol. 150, no. 1, pp. 140-167, 1943.

[21] H. Kimura, "Application of Orowan theory to hot rolling of aluminum," Journal of Japan Institute of Light Metals, vol. 35, no. 4, pp. 222-227, 1985.

[22] J. G. Lenard, F. Wang, and G. Nadkarni, "Role of constitutive formulation in the analysis of hot rolling," Transactions of ASME Journal of Engineering Materials and Technology, vol. 109, no. 4, pp. 343-349, 1987.

[23] D. J. Gates and T. Tarnopolskaya, "Linear theory for lateral displacements of a metal strip in a tandem cold-rolling mill with asymmetries," Proceedings of the Institution of Mechanical Engineers C, vol. 222, no. 7, pp. 1131-1148, 2008.

[24] S. Kikuchi, H. Kuwahara, N. Mazaki, S. Urai, and H. Miyamura, "Mechanical properties of Ag-Ni super-laminates produced by rolling," Materials Science and Engineering A, vol. 234-236, pp. 1114-1117, 1997.

[25] A. Atreya and J. G. Lenard, "Study of cold strip rolling," Transactions of ASME Journal of Engineering Materials and Technology, vol. 101, no. 2, pp. 129-134, 1979.

[26] S. Domanti and D. L. S. McElwain, "Two-dimensional plane strain rolling: an asymptotic approach to the estimation of inhomogeneous effects," International Journal of Mechanical Sciences, vol. 37, no. 2, pp. 175-196, 1995.

[27] A. G. Owen and A. W. J. Griffin, "Rapid solution of Orowan's equations using a hybrid computer," Proceedings of the Institution of Electrical Engineers, vol. 119, no. 10, pp. 1510-1516, 1972.

[28] D. Rusia, "Improvements to Alexander's computer model for force and torque calculations in strip rolling processes," Journal of Materials Shaping Technology, vol. 8, no. 3, pp. 167-177, 1990.

[29] T. A. El-Bitar, "Computer program for the calculation of roll force and torque with strip tension in cold rolling," Iron Steelmaker, vol. 20, no. 5, pp. 87-96, 1993.

[30] J. Yanagimoto, T. Morimoto, R. Kurahashi, and I. Chikushi, "Mathematical modelling for rolling force and microstructure evolution and microstructure controlling with heavy reduction in tandem hot strip rolling," Steel Research, vol. 73, no. 2, pp. 56-62, 2002.

[31] S. Alexandrov, G.-Y. Tzou, and M.-N. Huang, "Plane strain compression of a rigid/perfectly plastic multi-layer strip between parallel platens," Acta Mechanica, vol. 184, no. 1-4, pp. 103-120, 2006.

[32] S. Alexandrov, G. Mishuris, and W. Miszuris, "An analysis of the plane-strain compression of a three-layer strip," Archive of Applied Mechanics, vol. 71, no. 8, pp. 555-566, 2001.

[33] I. F. Collins and S. A. Meguid, "On the influence of hardening and anisotropy on the plane-strain compression of thin metal strip," Transactions of ASME Journal of Applied Mechanics, vol. 44, no. 2, pp. 271-278, 1977.

[34] T. Inoue, A. Yanagida, and J. Yanagimoto, "Finite element simulation of accumulative roll-bonding process," Materials Letters, vol. 106, pp. 37-40, 2013. 
[35] T. Inoue and N. Tsuji, "Quantification of strain in accumulative roll-bonding under unlubricated condition by finite element analysis," Computational Materials Science, vol. 46, no. 1, pp. 261-266, 2009.

[36] S. Alexandrov and N. Alexandrova, "On the maximum friction law for rigid/plastic, hardening materials," Meccanica, vol. 35, no. 5, pp. 393-398, 2000.

[37] S. Alexandrov and O. Richmond, "Singular plastic flow fields near surfaces of maximum friction stress," International Journal of Non-Linear Mechanics, vol. 36, no. 1, pp. 1-11, 2001.

[38] Y. Tomita and R. Sowerby, "An approximate analysis for studying the plane strain deformation of strain rate sensitive materials," International Journal of Mechanical Sciences, vol. 21, no. 8, pp. 505-516, 1979.

[39] N. Rebelo and S. Kobayashi, "A coupled analysis of viscoplastic deformation and heat transfer-II. Applications," International Journal of Mechanical Sciences, vol. 22, no. 11, pp. 707-718, 1980.

[40] E. J. Appleby, C. Y. Lu, R. S. Rao, M. L. Devenpeck, P. K. Wright, and O. Richmond, "Strip drawing: a theoretical-experimental comparison," International Journal of Mechanical Sciences, vol. 26, no. 5, pp. 351-362, 1984.

[41] R. E. Dutton, R. L. Goetz, S. Shamasundar, and S. L. Semiatin, "The ring test for P/M materials", Transactions of the ASME Journal of Manufacturing Science and Engineering, vol. 120, no. 4, pp. 764-769, 1998.

[42] S. M. Roberts, F. R. Hall, A. Van Bael et al., "Benchmark tests for 3-D, elasto-plastic, finite-element codes for the modelling of metal forming processes," Journal of Materials Processing Technology, vol. 34, no. 1-4, pp. 61-68, 1992.

[43] J. Helsing and A. Jonsson, "On the accuracy of benchmark tables and graphical results in the applied mechanics literature," Transactions ASME Journal of Applied Mechanics, vol. 69, no. 1, pp. 88-90, 2002.

[44] R. Sliwa, "A test determining the ability of different materials to undergo simultaneous plastic deformation to produce metal composites," Materials Science and Engineering A, vol. 135, pp. 259-265, 1991. 

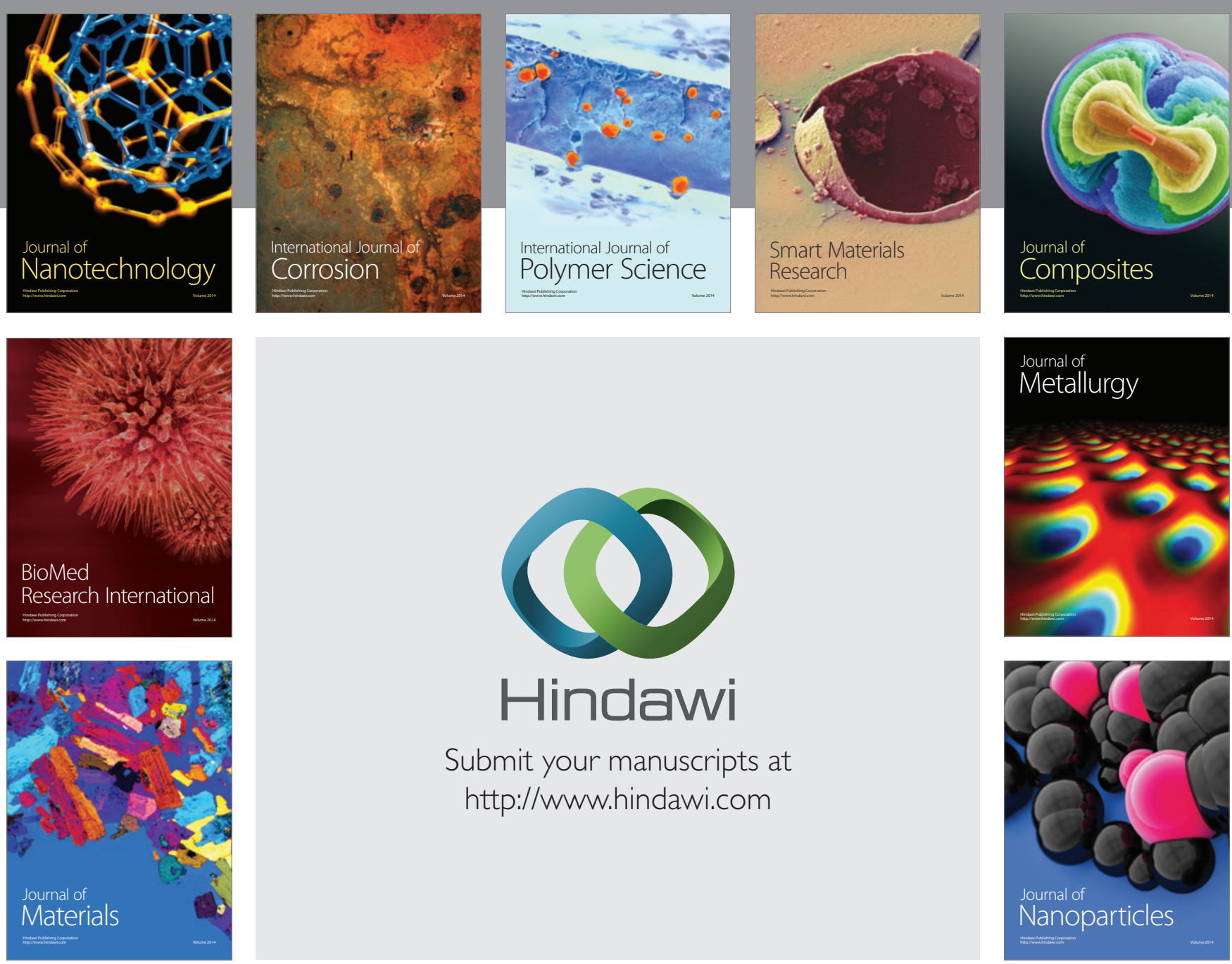

Submit your manuscripts at http://www.hindawi.com
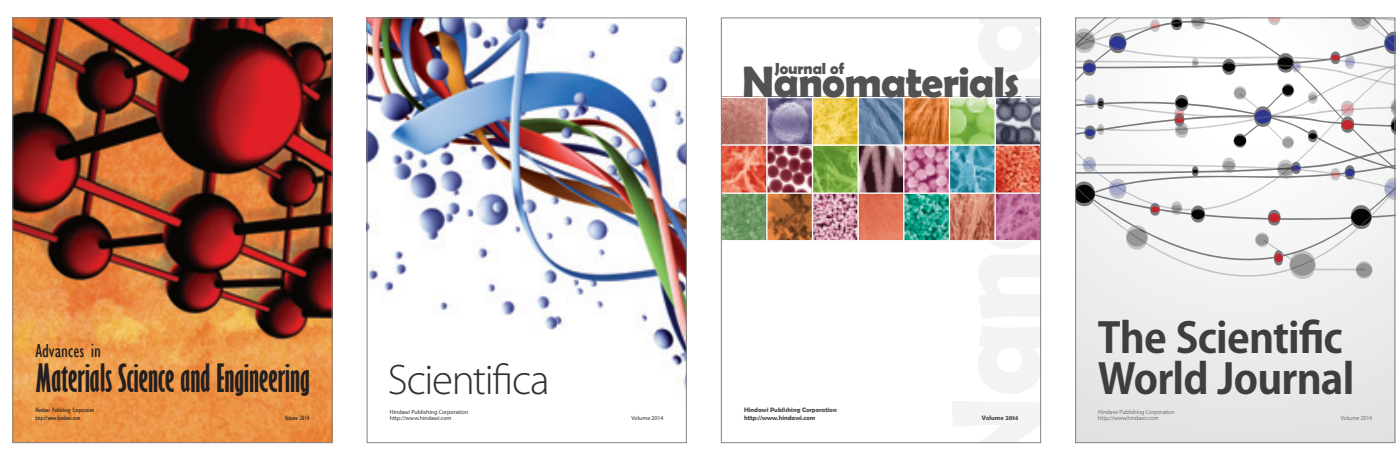

\section{The Scientific World Journal}
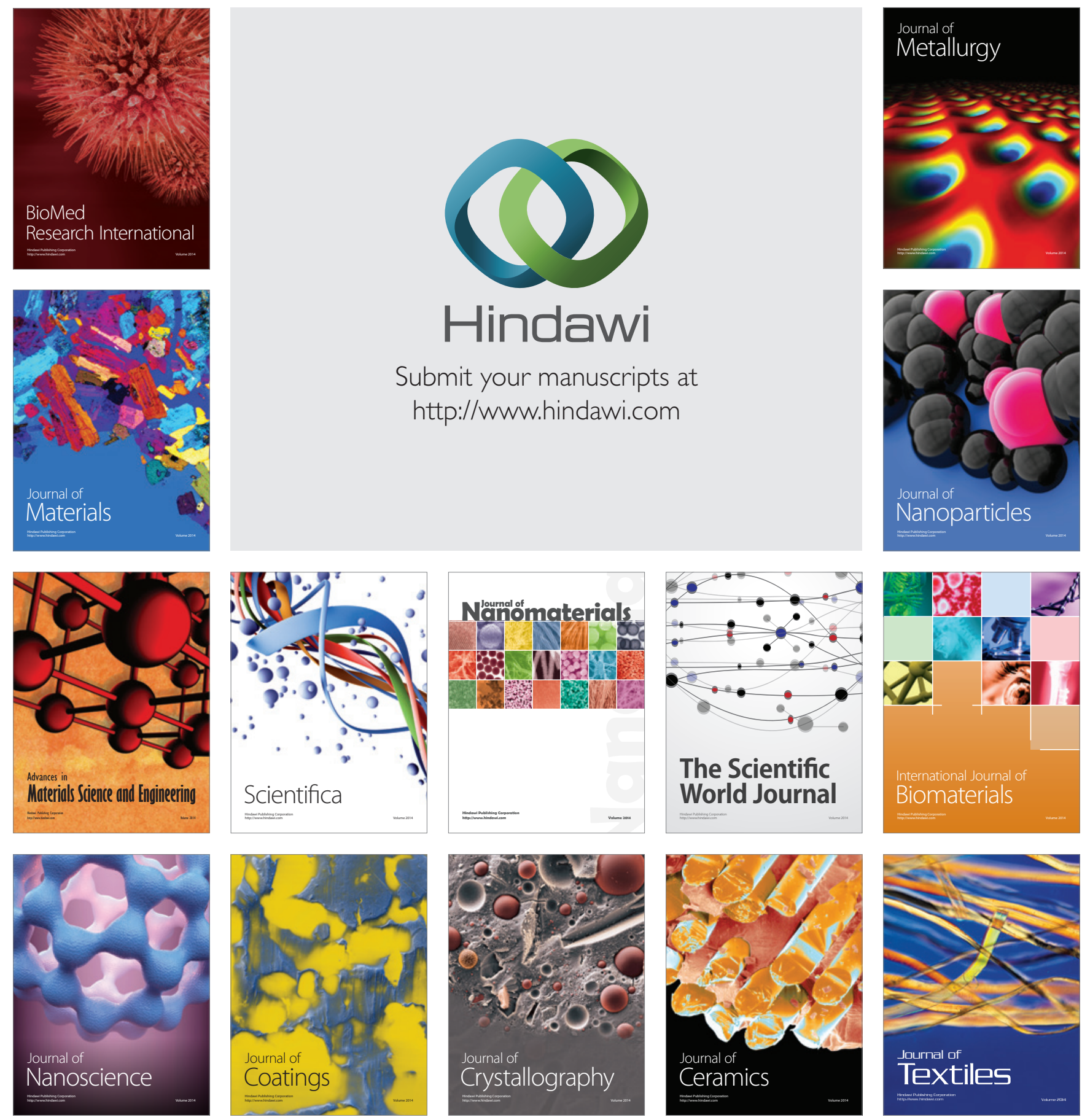